\title{
Device Fragments in Patient
}

National Cancer Institute

\section{Source}

National Cancer Institute. Device Fragments in Patient. NCI Thesaurus. Code C62946.

Issue associated with the inability to retrieve device and/or device fragments during medical procedures. 